\title{
Influencing factors analysis for high intensity focused ultrasound ablation in treatment of uterine fibroids.
}

\author{
Ji Yang, Gang Wei, Shuncang Zhang, Hui Zhang* \\ Department of Ultrasound, the Fourth Hospital of Xi'an, Xi'an, PR China
}

Abstract

\begin{abstract}
This study aims to analyse the influencing factors for High Intensity Focused Ultrasound (HIFU) ablation in treatment of uterine fibroids. Fifty-one uterine fibroids patients with 76 uterine fibroids were enrolled in this study. All patients received the HIFU ablation of uterine fibroids. The treatment efficacy was evaluated. The residual rate of ablation was calculated. The influencing factors related to the treatment outcome of HIIFU ablation were analysed. Results showed that, in 76 uterine fibroids, 25 $(32.9 \%)$ cases obtained effective treatment outcome, with residual rate $\geq 50 \%$, and $51(67.1 \%)$ cases obtained remarkably effective treatment outcome, with residual rate $<50 \%$. The treatment outcome had significant difference among subserous, submucous and intramura/ Ribro type $\left(\chi^{2}=6.614, P=0.037\right)$, among fibroid position of posterior wall, fundus uteri and anterio w $\left(\chi^{2}=11.410, P=0.003\right)$, between fibroid size of $<5 \mathrm{~cm}$ and $\geq 5 \mathrm{~cm}\left(\chi^{2}=4.259, \mathrm{P}=0.039\right)$, and amg 10 , equal and high contrast

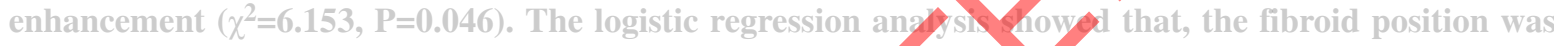
the independent risk factor of treatment outcome, with oddsatio o 2.545 (1.175-5.513). In conclusion, the fibroid type, fibroid position, fibroid size, and contrast enhacement are significantly related to the outcome of HIFU ablation in treatment of uterine filbroids. The fibroid position is the independent risk factor for treatment outcome.
\end{abstract}

Keywords: High intensity focused ultrasound, Ablaten, Mrluencing factors, Uterine fibroids.

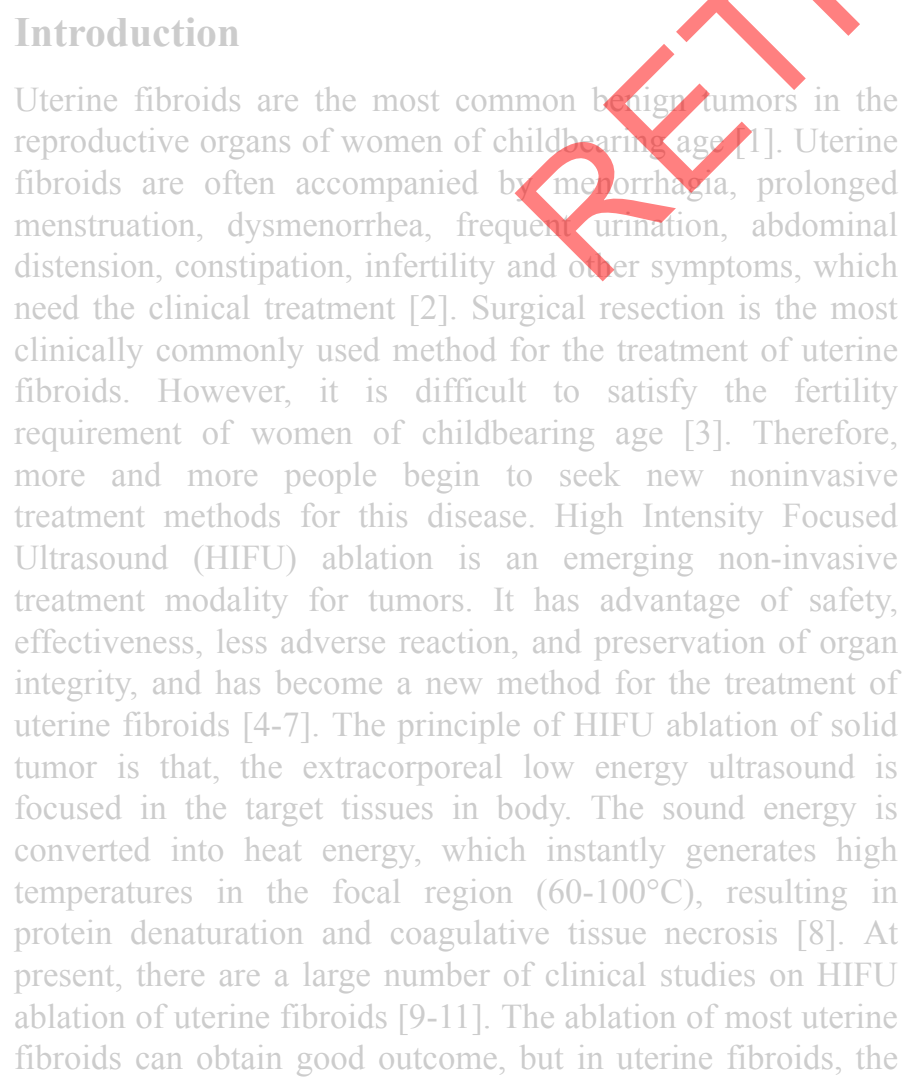

ablation rate is very low. There are many factors in pathological and ultrasound aspects which lead to different treatment outcomes. The present study systematically analysed the influencing factors for HIFU ablation in the treatment of uterine fibroids. The objective was to provide certain reference for the clinical application of HIFU ablation.

Patients and Methods

\section{Patients}

Fifty-one uterine fibroids patients with 76 uterine fibroids who were treated with HIFU ablation in The Fourth Hospital of Xi'an from May 2012 to January 2016 were enrolled in this study. The age of patients was 28-60 y old, with the average age of $42.3 \pm 5.4 \mathrm{y}$. The patients presented menorrhagia, prolonged menstruation, dysmenorrhea, frequent urination, abdominal distension and other symptoms. The uterine fibroids were diagnosed according to the clinical symptoms, gynecological examination and ultrasound or Computed Tomography (CT) examination. The inclusion criteria were as follows: i) the patients were diagnosed with uterine fibroids; ii) the patients had given birth, with no requirement of reproduction; ii) the diameter of fibroids was $2-8 \mathrm{~cm}$; iv) the patients had no artificial abortion within 3 months. The exclusion criteria were as follows: i) the clinical symptoms 